\title{
The role of qualitative research in clinical trial development: the EASE Back study.
}

Bartlam, B., ${ }^{1}$ Waterfield, J., ${ }^{2}$ Bishop, A., ${ }^{1}$ Holden, M.A., ${ }^{1}$ Barlas, P., ${ }^{2}$ Ismail, K.M., ${ }^{3}$ Kettle, C., ${ }^{4}$ Foster, N.E. ${ }^{1}$

${ }^{1}$ Arthritis Research UK Primary Care Centre, Institute of Primary Care and Health Sciences, Keele University, UK.

${ }^{2}$ School of Health and Rehabilitation, Institute of Primary Care and Health Sciences, Keele University, UK.

${ }^{3}$ Institute of Metabolism and Systems Research, College of Medical and Dental Sciences, University of Birmingham, UK.

${ }^{4}$ Faculty of Health Sciences, Staffordshire University, UK.

Corresponding author: Bernadette Bartlam: b.bartlam@keele.ac.uk

\begin{abstract}
This article outlines the rationale for adopting a mixed methods approach within randomized controlled trials (RCTs), and explores the associated challenges. Taking the example of the EASE Back feasibility and pilot study (Evaluating Acupuncture and Standard carE for pregnant women with BACK pain: ISRCTN49955124), we detail why and how we operationalized a concurrent-sequential mixed methods research design. We present findings from the exploratory research (focus groups and interviews with midwives and pregnant women), and explain how these were integrated with descriptive findings (a national survey of physical therapists) in order to inform and refine the design of the explanatory phase (the pilot RCT). We conclude with a discussion of implications for future mixed methods research design and conduct in RCTs.
\end{abstract}




\section{Keywords}

Pregnancy-related back pain, acupuncture, randomized controlled trials, mixed methods research, interdisciplinary health research.

\section{Introduction}

Within the field of health research the aim of a feasibility and pilot clinical trial is to assess the potential for the successful conduct of a main trial (Tickle-Degnen, 2013). The value of mixed methods in such preparatory work is increasingly recognized, and in particular the contribution of qualitative research in exploring uncertainties and identifying ways to optimize the successful delivery of more explanatory research phases, such as in the case of randomized controlled trials (RCTs) (O’Cathain, et al., 2015). However, adopting such a mixed methods approach can be challenging. Key to addressing such challenges is for researchers to present well-developed arguments for others to consider, articulating the basis on which they have proceeded (Christ, 2007; Tashakkori \& Creswell, 2007; Wisdom, Cavaleri, Onwuegbuzie, \& Green, 2012). Such transparency in reporting is increasingly recognized as critical in maximizing learning and improving evidence based research and practice (O’Cathain, Thomas, Drabble, Rudolph, \& Hewison, 2013).

The purpose of this article is to contribute to such an evidence base by presenting details of the concurrent-sequential mixed methods design adopted in the EASE Back study (Evaluating Acupuncture and Standard carE for pregnant women with BACK pain), a pilot RCT to establish the feasibility of a future, multi-center trial to investigate the clinical and 
cost-effectiveness of adding acupuncture to standard care for pregnant women with low back pain.

\section{Theoretical Background}

Three main areas of literature have informed our thinking around the relationship between qualitative research and clinical trials: first, theoretical considerations of how research questions predicate methods; second, debates around the challenges to inter-disciplinary teams using mixed methods in health research; and finally, the role and function of mixed methods, in particular exploratory qualitative research, in clinical trials. We now look at these in more detail and outline their methodological contribution to the EASE Back study.

\section{Research Questions and Methods}

Over the last two decades there has been a well-documented move away from seeing any one research method as the 'gold standard'. Instead, a more nuanced view has emerged which takes the research question as the driver for method (Mason \& Dale, 2011). Given that a strong mixed methods study starts with strong mixed methods research questions (Tashakkori \& Creswell, 2007), it is important to consider the types of questions being asked. Doing so ensures a robust design, and makes explicit the contribution of each type of question to the research study overall. Three typologies have been identified (Sim \& Wright, 2000): exploratory, where the topic may be relatively new or unexplored and the research question may change; descriptive where there is likely to be an outline body of knowledge lacking detail but the research question is quite specific; and explanatory where a well-defined body of work exists but with remaining questions which are framed in the form of an hypothesis. 
Each of these types of questions demands different methods, generating different forms of data (Johnson \& Onwuegbuzie, 2004). Exploratory questions generally require flexible and emergent methods, generating qualitative data; descriptive questions may be addressed through a fairly fixed and sequential design, and may produce both qualitative and quantitative data; and explanatory questions require a design that is highly structured and fixed from the outset, and will normally produce quantitative data (Sim \& Wright, 2000). Addressing two or more of these types of questions within one research study, and consequently mixing different methods, can generate findings leading to an understanding that is greater than the sum of the individual parts.

Adopting mixed methods "involves collecting, analyzing, and interpreting quantitative and qualitative data in a single study or in a series of studies that investigate the same underlying phenomenon" (Leech \& Onwuegbuzie, 2009, p. 267). Varying degrees of relationship and weighting between the methods have been identified, from the 'single method as dominant with additional data from other sources' through to the 'fully mixed, equal status' model (Leech \& Onwuegbuzie, 2009; Mason, 2006). When a study combines quantitative and qualitative data collection techniques to any degree, either it is using a fully mixed design or a partially mixed design; the key difference being that fully mixed methods designs involve mixing quantitative and qualitative techniques within, or across, one or more stages of the research process, whilst there is no mixing of techniques within or across stages in a partially mixed methods study (Leech \& Onwuegbuzie, 2009). In terms of analysis, three approaches have been identified: triangulation using different methods to gain a more complete picture; iteration by following a thread across analyses to identify themes and questions for further 
exploration; and a mixed methods matrix that focuses on single cases for which there are multiple data (O'Cathain, Murphy, \& Nicholl, 2010). EASE Back addressed all three types of research questions (exploratory, descriptive, and explanatory), analyzing data for triangulation in an iterative process. In doing so, it adopted a concurrent, sequential mixed methods design.

\section{Mixed Methods: Challenges in Health Research}

Interdisciplinary teams are most often characteristic of mixed methods research (Adler \& Stewart, 2010; Christ, 2007; Kessel \& Rosenfield, 2008). The need for such team working is increasingly recognized at a policy and research level, e.g. the European Union Research Advisory Board (2004), the Panel on New Directions in Social Demography, Social Epidemiology, and the Sociology of Ageing (2013) in the United States. Yet, there are considerable challenges for such teams working in the context of healthcare research, as McBride (2010, p. 76-7) notes:

"While all healthcare professionals have some training in interpersonal communication effectiveness, none of the professions is schooled sufficiently in the complex skills necessary to team building... There is widespread evidence that the healthcare professions do not know how to conduct crucial conversations with each other."

Key to developing such conversations is a shared paradigmatic understanding of methods, including their "epistemological, ontological, axiological, and philosophical foundations" (Freshwater, 2012, p. 3). However, many clinical researchers in healthcare come from professional backgrounds that offer little opportunity to consider such issues, resulting in 
potentially serious barriers to the development of the 'methodological bilingualism' necessary to successfully conduct mixed methods research (Curry et al., 2012). Consequently, the epistemological position and skills of lead researchers are crucial in creating an effective interdisciplinary culture, from supporting the identification of research questions through to fostering innovation in analytic techniques and developing shared writing approaches that are capable of integrating the different data, thus generating more detailed and comprehensive findings (Gray, 2008; Klassen, Creswell, Plano Clark, Smith, \& Meissner, 2012; O’Cathain, Murphy, \& Nicholl, 2008). Finally, it has also been argued that insufficient account is taken of the challenges around resources, including the time needed not only for data collection, but for analysis at a mixed methods level (Klassen et al., 2012). As we will explain later, consideration of these issues resulted in EASE Back adopting a study management structure that focused on cross-disciplinary inclusion and dialogue across all stages of the research, including time for inter-disciplinary analysis and interpretation of data.

\section{Qualitative Methods in Randomized Controlled Trials}

Whilst being costly and generally more time-consuming than other methods, an RCT is the most rigorous way of determining whether a cause-effect relationship exists between treatment and outcome, and for assessing the cost-effectiveness of a treatment (Moher et al., 2010). However, there is a growing awareness of the need to develop a more methodologically sophisticated approach to RCTs for a variety of reasons, including concerns over the quality and reliability of data, recruitment challenges, resource allocation needs, and the identification of future research priorities (Bartlam et al., 2012; Cherubini et al., 2011; 
Ross et al., 1999). In its guidance for complex interventions, the United Kingdom's (UK) Medical Research Council (MRC) (2008, p.4) notes that:

"All of the stages are important, and too strong a focus on the main evaluation, to the neglect of adequate development and piloting work, or proper consideration of the practical issues of implementation, will result in weaker interventions, that are harder to evaluate, less likely to be implemented and less likely to be worth implementing."

The MRC, together with the National Institute for Health Research (NIHR), are the two largest public funding bodies of RCTs in the UK. Many of the trials they fund struggle to recruit to their target sample size, and both time and financial extensions are often requested (McDonald et al., 2006; Sully, Julious, \& Nicholl, 2013). Moreover, evidence from the United States shows striking disparities in RCT participation with, in particular, women and minority ethnic patients more difficult to recruit to trials, where they are significantly underrepresented (Coakley et al., 2012). RCTs that incorporate qualitative approaches have potential value by optimizing the intervention content, delivery and acceptability, making trial recruitment and conduct more acceptable for participants and more efficient, facilitating interpretation of the findings, helping trial teams to be sensitive to the needs of participants, and saving resources by directing researchers towards interventions more likely to be effective in future trials (Donovan et al., 2002; Moore et al., 2015; O’Cathain et al., 2013). Finally, whilst the term 'clinical trial' is one that is commonly found in the media, it is in fact a highly complex research method that can be poorly understood by lay participants. This gives rise to concerns about the extent to which participants in trials give informed consent (Behrendt, Gölz, Roesler, Bertz, \& Wünsch, 2011; Hereu et al., 2010). Qualitative research can improve the quality and accessibility of participant information, as well as aid in the process of ensuring consent remains in place as the trial proceeds (Bartlam et al., 2012). These issues add to the growing call to move away from the inappropriate use of pilot trials 
as hypothesis testing to a greater emphasis on their descriptive, feasibility potential (Arain, Campbell, Cooper, \& Lancaster, 2010).

However, notwithstanding these arguments, and whilst their use has increased over the last decade, mixed methods using qualitative approaches remain relatively rare in RCTs. Where they are used, they are frequently under-reported (Lewin, Glenton, \& Oxman, 2009), with only a few best practice examples existing (Howard \& Howard, 2012). In their systematic mapping review, O'Cathain and colleagues (2013) could identify only $28 \%$ of RCTs between 2008 and September 2010 ( $n=82 / 296)$, reported in English, which described the use of qualitative methods. Furthermore, where they were reported, the value of the qualitative work to the RCT was not always made explicit. Part of the problem is the continued reluctance of high-impact medical journals to publish qualitative and/or mixed methods research reports (Greenhalgh et al., 2016). Reviewing the empirical research articles published between 1999-2008, Gagliardi \& Dobrow (2011) found that qualitative articles made up between $0 \%$ and $0.6 \%$ of articles published in general medical journals, and between $0 \%$ to $6.4 \%$ of those in the health services and policy research journals. One consequence is that findings from mixed methods studies are most often presented in separate parallel publications (Bryman, 2007), with a lack of incentive for researchers to spend time integrating data at the analysis stage (O'Cathain et al., 2013).

With these various concerns in mind, EASE Back was designed in two phases, with phase one integrating findings from the exploratory qualitative data and the descriptive survey data to inform phase two, the feasibility pilot RCT.

\section{The EASE Back Feasibility and Pilot RCT}


The rationale for EASE Back was based on a review of the literature which indicates that that low back pain (LBP) during pregnancy is very common and is estimated to affect between $45 \%-75 \%$ of women at some stage during their pregnancy (Pierce, Homer, Dahlen, \& King, 2012; Wu et al., 2004), that pregnant women with LBP have lower quality of life compared with those with no LBP (Olsson \& Nilsson-Wikmar, 2004), and that between 20\%$23 \%$ of women take sick leave because of their pain (Pierce et al., 2012).

No high quality UK data are available describing the proportion of women who currently receive treatment for pregnancy-related LBP. However, in an Australian study, $71 \%$ of women reported their problems to their maternity carer but only $25 \%$ received any treatment (Pierce et al., 2012). There is some suggestion that this may be related to healthcare professionals' lack of knowledge about available treatments, and fear of possible harm to the developing fetus (Vermani, Mittal, \& Weeks, 2010). The use of acupuncture for musculoskeletal problems in general is increasing and it is recommended within UK national guidelines for the management of persistent non-specific LBP, although not specifically in the context of pregnancy (National Institute for Clinical Excellence, 2009). A recent UK survey concluded there is wide variation in acupuncture practice (Bishop, Zaman, \& Lewith, 2011) and, with specific reference to pregnancy, to-date two systematic reviews have been published that have evaluated acupuncture treatment for this population. Both found acupuncture and stabilizing exercises relieved pain more than usual prenatal care, and acupuncture provided more relief from evening pain than exercise (Ee, Manheimer, Pirotta, \& White, 2008; Pennick \& Liddle, 2013). Furthermore, no major adverse events have been reported on the pregnancy, mother, labor and/or the fetus/neonate, even when acupuncture was administered with a stimulation that could be considered strong (Elden, Ostgaard, Fagevik-Olsen, Ladfors, \& Hagberg, 2008). 
Overall then, there is limited though promising evidence for the safety and effectiveness of acupuncture for LBP in pregnancy. However, there are no high quality trial data with long term follow-up regarding its clinical or cost-effectiveness in comparison to standard care and, although previous work identified acupuncture as acceptable to patients with non-specific LBP (Thomas et al, 2005), little is known about its acceptability amongst pregnant women, midwives and/or physical therapists.

\section{Study Aim and Design}

The aim of the EASE Back study was to explore the feasibility of a future main RCT testing the additional benefit of acupuncture to standard care in women with pregnancy-related LBP. The research questions fell into all three categories of questions highlighted previously: exploring the unknown views and experiences of women and health care practitioners; describing physical therapy current practice; and explanatory (hypothesis testing RCT of acupuncture for LBP in pregnancy). The study was designed in two phases: an exploratory qualitative enquiry running concurrently with a descriptive national survey, with the integrated findings from both sequentially informing the explanatory pilot RCT, see Figure 1. We now focus on the concurrent methods in phase 1, and explain how exactly these informed the pilot RCT (phase 2).

Figure 1 somewhere here: Question-driven mixed methods process model in EASE Back

\section{Concurrent Methods}

The concurrent methods had four objectives:

1. To identify standard care and acupuncture practice for pregnancy-related LBP. 
2. To explore the views of pregnant women with LBP about the acceptability of the proposed trial interventions, the content and mode of delivery of participant information, the most important outcomes, and the most appropriate timing of outcome measures.

3. To investigate the views of midwives and physical therapists regarding the acceptability and feasibility of acupuncture for women with pregnancy-related LBP.

4. To explore the views of midwives and physical therapists on the proposed feasibility of the pilot trial design, processes and interventions.

\section{Descriptive Quantitative Data Collection}

A survey was designed to describe standard care for pregnancy-related LBP and current practice of acupuncture, and was posted to 1093 physical therapists working in the UK, across specialty areas of musculoskeletal, acupuncture and women's health. The survey captured demographic and practice data, and explored the management of pregnancy-related LBP using a case vignette and associated clinical management questions.

\section{Exploratory Qualitative Data Collection}

To address the other three objectives, exploratory methods were adopted. These consisted of qualitative focus groups or individual interviews (in person or by telephone) with pregnant women, midwives and physical therapists. Since both women (Coakley et al., 2012) and healthcare practitioners (Wilkinson, Powell, \& Davies, 2011) can be difficult to engage in research, participants were offered a choice of interview format. This was a pragmatic decision rather than a theoretically driven one, and intended to meet the needs of participants in terms of convenience. All participants were given full information about the study ahead of deciding to participate. The midwives and physical therapists were invited to complete a 
brief questionnaire to describe their qualifications and experience, and the women completed a brief socio-demographic profile questionnaire.

Semi-structured interview guides were developed from the research objectives. The interviews with the women explored their experiences of LBP; its impact on their quality of life and activities of daily living; the care or support they felt they did, or did not, receive from family, friends and work colleagues; and their expectations post-pregnancy. The questions to the midwives and physical therapists focused on their experience of LBP amongst patients and the ways in which they managed symptoms; and their views on managing this patient population, in particular any difficulties. In addition, all participants were invited to share their views on a range of related issues, including complementary therapies generally and acupuncture specifically, particularly in relation to its use in pregnancy; their understanding of, and views on, research generally; the sort of information that might be required to reach a decision around participation in an RCT; the most important outcomes to measure, and the most appropriate timing of those outcomes.

Two members of the research team facilitated the focus groups. The steps outlined in the participant information leaflet (PIL) on data anonymity and participant confidentiality were highlighted and consent checked. In line with Kitzinger (1994), participants were reminded of the importance of valuing the views of others, even when these might not be shared, and that the aim was to capture as wide a range of perspectives as possible. All interviews and focus groups were audio-recorded with consent. Data collection with all three sets of participants took place concurrently from June 2012, and ceased when data saturation was reached. 


\section{Recruitment}

In the planned recruitment period for women to the qualitative research it was anticipated that there would be 600 women with pregnancy-related LBP who could be invited to participate. This was the number of pregnancies overseen by the participating maternity center in a fourmonth period. A convenience sampling strategy (Morse, Barrett, Mayan, Olson, \& Spiers, 2008) was adopted and any woman with pregnancy-related LBP could either self-refer, or agree for her healthcare practitioner to pass on her contact details to the research team. A flyer and poster were designed outlining the study and providing contact details.

In total 3,000 flyers and 100 posters were distributed through various means: general information packs when the woman first booked in; local ante-natal clinics; community midwives giving the flyers directly to pregnant women under their care; the women's health physical therapy service 'back class' for pregnant women at the local hospital. An invitation to participate was also posted on internet sites such as Mumsnet (http://www.mumsnet.com) and the Pelvic Pain Support Network (http://www.pelvicpain.org.uk).

When contact details were received by the research team, the women were telephoned to check if they were still willing to be interviewed, and if so, to arrange a convenient time. At that point, a letter confirming the arrangements, the PIL and two copies of the consent form, together with a stamped-addressed envelope for the return of a signed copy of the consent form, were posted out. The PIL and consent form were discussed in detail at the time of the interview to check for understanding, and consent was also audio recorded. A total of 17 women were interviewed. 
For the midwives and physical therapists, a purposive sampling strategy was adopted to ensure a range of experience and perspectives. Two teams of community midwives were approached, together with the group of research midwives working in the participating maternity hospital and who would be recruiting women for the pilot RCT, giving a total of three focus groups. All members of the teams were willing to take part and the focus groups were arranged to fit with their regular team meetings. Only those who were on leave did not take part. A further three focus groups were arranged with the physical therapists from the local community musculoskeletal outpatient service and from the local hospital women's health physical therapy service, who would be delivering the interventions in the pilot RCT. The invitation was issued through the service managers who were aware that between five and ten people were needed for each of the groups. In addition, a sample of those physical therapists $(n=30)$ that consented for further contact on returned questionnaires from the national survey were also invited to take part. Because of geographical spread and participant convenience, these individuals were interviewed by telephone. The interviews took place after the focus groups with physical therapists and, because of data overall saturation, were limited to three individuals. In total, 15 midwives and 21 physical therapists took part in the exploratory research, giving a total of 53 individuals (Table 1).

Table 1 somewhere here: summary of interview and focus group participants

\section{Analysis}

The responses to the survey and the profile questionnaires completed as part of the interviews/focus groups were analyzed descriptively. An exploratory thematic analysis was adopted for the qualitative interviews and focus groups, within a constructivist grounded theory framework; emergent findings were checked out in subsequent interviews across all 
three groups of participants, in an iterative cycle (Charmaz, 2006). All discussions were digitally recorded, lasted 20 to 60 minutes and were transcribed. To preserve participants' anonymity, all were given unique ID numbers. To maximize the benefits of being an interdisciplinary team, the coders brought differing disciplinary perspectives to bear on the qualitative data (Bartlam social science; Barlas acupuncture; Waterfield physical therapy). To ensure inter-coder reliability, each independently coded a random selection of interviews as part of agreeing the coding frame, which was then applied across the whole data set, checking for consistencies and confounding cases. As a further form of triangulation and in order to integrate findings (Miles and Huberman, 1994; Leech \& Onwuegbuzie, 2009; O’Cathain et al, 2010), data from the interviews and focus groups were also compared for differences and similarities with the descriptive survey responses on the experience in treating, and management of, the condition. The results across the two methods were found to be convergent (O’Cathain et al. 2010).

\section{Findings}

\section{The Descriptive Survey}

The response rate to the national survey was $57.5 \%$ ( $n=629)$, with responses from 499 physical therapists experienced in treating pregnancy-related LBP included in the analysis. A total of 16 advice and 18 treatment options were reported for the management of the patient vignette. Most frequently reported were: advice on posture (98\%) and work (88\%), the use of home exercise programs (94\%), postural exercises (93\%), support belts (48\%), and manual therapy (48\%). Use of acupuncture was reported by $24 \%$ of respondents. A typical course of treatment for women with pregnancy-related LBP was two to four face-to-face treatment sessions over six weeks. Respondents were also asked whether they routinely used specific advice or self-management leaflets in the management of pregnancy-related LBP, and if so, 
to enclose a copy of the leaflet with their response. This resulted in 37 different advice and self-management leaflets. Full details of the findings from the survey are reported elsewhere (Bishop, Holden, Ogollah, \& Foster, 2015).

The Exploratory Qualitative Interviews and Focus Groups

\section{Participants}

Of the 17 women interviewed, the average age was 26 years (range 22-34 years). Gestation ranged from 15-39 weeks, with a mean average of 32 weeks. For eight women it was their first pregnancy. In terms of ethnicity, eight described themselves as English, five as 'other British', three as 'other White', and one woman as 'African'. All were either married or living with a partner. Employment ranged from healthcare practitioners through to clerical workers.

Of the 15 midwives, the average length of practice was 18 years, with the majority $(n=9)$ qualified for over 12 years. The least experienced person had been qualified for three years, and there were also two maternity assistants included in the focus groups. None reported any specific postgraduate training about LBP in pregnancy. Six midwives reported seeing pregnant women with LBP either very frequently (at least one per week) or frequently (at least one per month). Just one midwife reported seeing such patients infrequently (at most one in the last six months).

As with the midwives, the 21 physical therapists were experienced practitioners. Their average length of practice was 12 years; nine had been qualified for 12 years or more (one for 
36 years) and the least experienced person had been qualified for over three years. In terms of contact with pregnant women, seven reported seeing such patients infrequently. All of these were community-based physical therapists, and all apart from one reported that they had no specific postgraduate training about pregnancy-related LBP. Unsurprisingly, the women's health physical therapists leading the hospital based educational 'back class' reported seeing such patients frequently (full details of the physical therapists views and the broader implications for education and training are reported elsewhere (Waterfield, Bartlam, Holden, Bishop, Barlas, \& Foster, 2015).

Three key themes emerged from the data across all three groups: the high burden of LBP in pregnancy; the paucity of treatment options; and the acceptability of acupuncture. Below we present details of these, before turning to examine their methodological implications for the pilot RCT.

High Burden of LBP in Pregnancy: "In the morning when I get up, I kind of crawl out of bed...”

The high burden of pregnancy-related LBP first became evident from the difficulties in recruiting the women. Despite the plethora of flyers, posters, efforts on the part of clinical staff to discuss the study with potentially eligible women, and extending the recruitment period of the interviews by a further two months, only 43 women agreed to contact from the research team - a response rate of only $7 \%$ of all those eligible. Of these, two self-referred, two were referred by physical therapists, three by obstetricians, and 20 were referred to the research team by community midwives. The remaining 16 were identified through members 
of the research team who attended the educational back class within the women's health physical therapy service in the local hospital (see Table 2). No responses were received to the online invitations.

Table 2: Summary of sources of interview referral of women somewhere here.

There were also challenges in making contact with the 43 women who expressed an interest. On average, it took five phone calls spread over different times of day, including the evenings, to make initial contact. Two people refused to participate in the interviews when contacted: one no longer had LBP and the husband of the other was ill. One woman was subsequently unavailable at the time of the agreed interview. Twenty-three women were not contactable within the five contact attempts. The difficulties in making contact were taken to the midwives and physical therapists for advice, who expressed no surprise and attributed the difficulties to a client population struggling to cope with everyday life. Moreover, they suggested that travelling to a focus group meeting would be an additional and unacceptable burden for these women. This was borne out in the recruitment telephone calls where the women consistently opted for telephone interview. A total of 17 women were interviewed over the six-month recruitment period, representing $39 \%$ of those who consented to further contact. Fortunately, despite these difficulties, as the characteristics of the women suggest, the sample was diverse and the qualitative data were rich and sufficient for data saturation.

During the interviews with women a picture of the burden of pregnancy-related LBP, and it's often wide-ranging impact on activities of daily living, emerged strongly. For those in severe 
pain, activities of daily living were difficult, leaving them unable to work or participate in social activities. Most reported good support from partners and family. However, there were also reports of little support, particularly in the workplace, with colleagues seeing LBP as a normal part of pregnancy and expecting women to "just get on with it". Women described difficulties in negotiating time from work to attend even routine antenatal appointments and considered having time for anything that might incur further absence, such as research participation, yet more difficult.

Paucity of Treatment Options: "There's very little to offer'

The responses to the survey indicated that standard care varies widely for this patient group (Bishop et al., 2015). This was reflected in the experiences of the women and professionals, who highlighted the paucity of treatment options. Midwives and physical therapists reported explaining the causes of LBP during pregnancy as a way of reassuring the women and, although they described offering advice, they felt that this amounted to 'fobbing-off' their patients, reflecting their lack of faith in the effectiveness of their own suggestions. Moreover, the advice provided was highly variable, indicating uncertainty regarding the most appropriate guidance to offer, and no sources of advice, either in terms of written leaflets or website resources, were used with any consistency, as underlined by the 37 different leaflets returned by physical therapists with their survey responses. Most women were advised to try self-management techniques around posture, gentle exercise, and pain relief positioning and medication. The physical therapists favored a 'hands off' approach, providing reassurance and giving advice on posture, preparation for labor and delivery, and feeding positions after delivery. The uncertainty about what constituted 'the right advice' for this group of patients was also reflected in the accounts of the women, who reported being left to 'get on with it'. 
Acupuncture for Pregnancy-Related LBP: "Something to alleviate it for a little bit".

Although some women did express the need for information and reassurance over the safety of acupuncture, and whether positioning for acupuncture would require them to lie down in ways that could exacerbate their pain, in the main there was very little concern about its use in pregnancy. In terms of the timing and measurement of outcomes if they had acupuncture, women said they would not expect to experience an immediate difference but would anticipate doing so over three to four treatments. For actual outcomes, and perhaps reflecting the paucity of support they experienced, expectations were very modest; women felt that it was unlikely the pain could be completely resolved but that any degree of alleviation, however limited, would be acceptable and was seen as a realistic outcome.

Findings from the discussions with the midwives very much reflected the views of the women. They were in favor of acupuncture as a useful additional source of help for the pain; felt that many women would be interested in knowing more about it, particularly where their pain is severe; that women would be willing to try it within the context of a trial; and that women would have few concerns and where they did, these would be most likely linked to the positioning of, and sensations from, the needles.

Whilst the physical therapists were also in favor of trialing the additional benefit of acupuncture for this patient group, they raised concerns about its safety in pregnancy. Such concerns - consistent with the findings from the survey - included issues around general safety and specific acupuncture points and techniques to be used. Indeed for most, acupuncture was viewed as contra-indicated in pregnancy. It was clear that these concerns were rooted in a lack of confidence and/or experience in treating pregnant women. However, 
although the majority view, this were not shared by all, as evidenced by the three physical therapists from the national survey who were confident about the safety and efficacy of acupuncture for this population, and indeed considered it safer than medication. Echoing the importance of practical experience and confidence, all three were acupuncture trained and all worked in NHS musculoskeletal outpatient departments where acupuncture was freely available for pregnant women. One had been qualified for 11 years, another for 18 years and one for 28 years.

\section{Implications for the Explanatory Pilot RCT}

These findings had clear implications for three key methodological issues in the subsequent pilot RCT: recruitment; development and delivery of the standard care intervention; and the training program for physical therapists delivering the interventions.

\section{Recruitment}

It was anticipated that women would be identified for the pilot RCT through community midwives, obstetricians and antenatal clinics within the participating maternity center. However, the challenges in recruitment to the qualitative work through these routes pointed to the need to develop and test a broader range of recruitment strategies for the RCT. The findings of the interviews, in particular those with the midwives, led to the development of several additional methods: a brief questionnaire screening on LBP for all pregnant women attending their routine antenatal 20 week ultrasound scan appointment that included asking whether they would be willing to be contacted further; screening patients referred to the women's health physical therapy service at the participating hospital to identify pregnant women with LBP; a local awareness raising campaign was developed that included a study website, a YouTube video, advertisements placed in a local newspaper, local radio stations 
and on local buses in order to take the message about the study directly to local pregnant women who could then opt to self-refer to the research team for eligibility screening.

Furthermore, the original intention had been that research midwives would screen all women for eligibility in face-to-face meetings. Through the focus group discussions with midwives and the interviews with the women, it became clear that a much more acceptable approach for pregnant women, and a more efficient use of research midwives' time, would be to conduct a brief telephone screening first and only invite those who appeared potentially eligible to faceto-face meetings for full eligibility screening, informed consent and baseline data collection.

Underpinning these various recruitment approaches was a portfolio of documents developed in the light of the qualitative findings, including flyers, posters, and a study 'business' card with contact details. In order for potential participants to be fully informed about what taking part in the pilot RCT would involve, a detailed PIL was developed. The PIL was based on both a best practice example provided within the Good Clinical Practice and Regulatory Requirements for Clinical Trials (http://www.cf.ac.uk/racdv/resgov/Resources/013595en.pdf), and from an analysis of the issues raised in the interviews and focus groups. Consequently, it addressed questions about the nature of acupuncture needles, any known risks to the mother or baby, positioning and time for treatment. Information was provided on the rationale for the study, why women were being invited to take part, what taking part would involve, issues around anonymity and confidentiality, payment, and details of the funding source of the trial. Patient members of the Trial Steering Committee (TSC) reviewed the draft and some of the language was amended to be more accessible to a lay reader. In addition, several options were offered to the women 
in terms of timing and location of treatments, including the provision of early evening appointments over a large geographical spread within the local area.

Over its six months duration the pilot RCT recruited $45 \%$ of potentially eligible women, sufficient to meet its key objectives in providing reasonable estimates of recruitment and retention rates and potential treatment effect.

\section{Development of the Standard Care Intervention in the Pilot RCT}

The concern was to ensure that the intervention protocol for standard care reflected the best of care currently available in the UK, and thus could provide a fair comparison in the RCT. As a result of the qualitative findings highlighting a dearth of information and advice for these women, a high quality and very comprehensive self-management booklet entitled 'EASE Back: Managing your back and pelvic girdle pain in pregnancy' was developed. This explicitly addressed the issues that emerged in the qualitative data. In addition, the best information from the 37 leaflets returned through the survey was also integrated into the study booklet. To maximize its usefulness and to ensure that it had sufficiently captured the breadth of experience needed, the booklet also had input from a wide range of other individuals, including specialist women's health physical therapists, a qualified antenatal exercise teacher and patient members of the TSC. The booklet contains wide-ranging advice on exercises, posture and movement, adaptations in lifting/moving, pelvic and back support aids, pacing activities and rest, pain medication, managing work and daily activities, information on how to access physiotherapy and reassurance about the likelihood that the pain would resolve when the pregnancy ended. It provides information in an accessible and straightforward style, using photographs of a pregnant woman to illustrate examples of 
specific adaptations to posture, etc. To increase accessibility yet further, it was produced by a professional graphic design company.

For those within the standard care arm of the RCT who had severe pain, and who felt they needed more than the booklet, a pathway to one-to-one physical therapy (of between two to four treatment visits) was also available. The content of this was also informed by a combination of the findings from the qualitative research and survey findings, and consisted predominantly of advice and exercise interventions, but also the use of support maternity belts, manual therapy and massage.

\section{Training Program for Participating Physical Therapists}

A total of 14 physical therapists were trained to deliver the interventions in the pilot RCT. The training was delivered over three and a half days. The original proposal anticipated that this would focus on the acupuncture intervention protocols for pregnancy-related LBP, including parameters of stimulation recommended for optimal pain relief, and include demonstration and practice of the intervention. In addition, it was to include discussion of how to work to an intervention protocol in a RCT, and review and reach agreement about the case report forms to be used to record treatment details.

However, in the light of the findings from the qualitative research, an emphasis was included to encourage physical therapists to voice any concerns about acupuncture or its safety so that these could be addressed openly. To facilitate this, the final program was developed in line with Bloom, Engelhart, Furst, Hill, and Krathwohl's (1956) taxonomy, that is, ensuring that 
the affective domain (attitudes and emotions) was covered in addition to the cognitive (intellectual) and psycho-motor (physical skills) domains. In practical terms this resulted in a multi-method training approach combining didactic lectures, group discussion, real case examples and skills practice (with pregnant women who offered to be models for the training) that focused on cognitive and physical skills of all aspects of assessment of patients, standard care and acupuncture intervention protocols. In addition, to address the affective domain and reassure the physical therapists and build their confidence, existing good quality evidence on the safety of acupuncture identified in the literature review was presented in detail and discussed. The physiology of pregnancy was reviewed and specific sessions with the consultant obstetrician member of the team (Ishmail) were included to discuss pregnancy complications and their severity and risk. Finally, a process of on-going mentoring and support for participating physical therapists was adopted.

\section{Discussion}

EASE Back offers an example of how qualitative research can contribute to the effective preparation for an RCT, in this case by sensitizing the research team to the needs of participants and collaborators, resulting in more effective trial recruitment methods and conduct. In what follows we discuss how some of the challenges associated with mixed methods research in health referred to earlier were addressed in this study.

First, we would agree that building a strong team is critical, with shared goals and a culture that supports consensus building across all aspects of the work, fostering the necessary methodological bilingualism needed. In terms of how such collaboration was operationalized in EASE Back, the study team comprised researchers with backgrounds in acupuncture, 
biostatistics, health economics, midwifery, obstetrics, physical therapy, social science and trial management. There were regular monthly team meetings over two years to which all members of the team were expected to attend and contribute. On rare occasions teleconferencing was used but in-person attendance was generally excellent. All members of the team were included in the development and refinement of all aspects of the research, and comments and input encouraged by the different method leads (Bartlam [qualitative], Bishop [survey], Foster [trial]). Meetings were chaired in such a way that the views of each individual were actively sought and received constructively. Whilst some items featured more prominently at particular moments in the research process, there was a standing agenda that included all aspects of the research. Bartlam carried out all the individual interviews. However, she co-facilitated each focus group with a different member of the research team. This enabled other members of the team to gain an immediate sense of the challenges that focus group participants were anticipating might be encountered in the pilot RCT, and also to appreciate how these were reflected in the interview data with the women. Involving different disciplinary perspectives in the analysis was critical to ensuring robust interpretation of the data. Emerging findings from the qualitative research were regularly presented to the full team, and divergent explanations were considered. It was during the team meetings that the discussions around how these did or did not reflect the findings from the survey took place. This triangulation increased confidence about integrating the findings into the pilot RCT. The wider team took responsibility for identifying strategies to address the challenges identified through the emerging findings, i.e. for recruitment and training, ensuring no delays to the pilot RCT. The TSC, in addition to consisting of external independent experts advising on the conduct and progress of the study (see https://www.mrc.ac.uk/documents/pdf/goodclinical-practice-in-clinical-trials/), also included lay members who were able to offer advice on emerging findings from the exploratory research and comment on participant facing 
documents, etc. The Patient and Public Involvement and Engagement (PPIE) in EASE Back was showcased by the NIHR as an example of good practice (http://www.invo.org.uk/wpcontent/uploads/2015/10/NETSCC-PPI-impact-case-study3final.pdf)

Second, we support the argument that the culture of collaboration necessary for effect interdisciplinary mixed methods will struggle to exist without broader, strategic support structures. The EASE Back study team sits within the Research Institute for Primary Care at Keele University, UK. Within the Institute there is a strong emphasis on interdisciplinary collaboration and developing the necessary methodological bilingualism to support the use of mixed methods where appropriate. In addition to a multidisciplinary staff group that includes epidemiologists, primary care physicians, occupational therapists, nurses, physical therapists, psychologists, rheumatologists, social scientists and statisticians, methodological expertise ranges from a variety of longitudinal and cross-sectional research approaches including population surveys, clinical trials and a wide range of qualitative methods. Staff are expected to develop research proposals in collaboration with a range of colleagues, including patient and public representatives.

Third, EASE Back highlights the importance of adequate resources, in particular time, for qualitative methods. The difficulties in recruiting to the pre-trial qualitative work, and the unanticipated extra time and effort that went into addressing that, could have been read as a failure in methods and left there. Without the detailed and time-consuming attention to exploring and understanding the data in terms of implications for recruitment strategies, information/support and training, it is highly likely that the pilot RCT in turn would have struggled to recruit participants. Such a far-reaching contribution was only possible because 
of the degree of methodological bilingualism, and trust, within the research team, and the time given to the qualitative work.

Finally, we feel that there are lessons to be learned from EASE Back about dissemination and increasing the impact of findings from mixed methods research generally. Given the word count of most healthcare journals - typically around 5000 - the findings from EASE Back are reported in a range of publications in addition to the present one: the national survey (Bishop et al., 2015); physical therapists views on the role of acupuncture in pregnancy and implications for training and practice (Waterfield et al., 2015), the EASE Back final report (Foster et al., 2016) and the pilot RCT paper (Bishop et al., under review 2016). Where possible these cross-reference the others but it has not been possible to do so when articles such as this were still in preparation at the time of publication of the first, i.e. the Bishop et al., (2015) and Waterfield et al., (2015) articles do not reference this article. Whilst an emphasis on being concise is clearly appropriate, in the present digital age, we would suggest that word counts per se are increasingly irrelevant - not least because of the limits they impose in reporting integrated findings from mixed methods research, as our experience indicates. A consideration of a more flexible approach on the part of health science journals would appear warranted.

\section{Conclusions}

Integrating the exploratory qualitative findings with those of the concurrent descriptive survey in EASE Back helped refine the design and processes of the successful pilot RCT. Whilst the case for the development and piloting of RCTs has been well argued (MRC, 2008), the contribution of qualitative methods in this study point to the importance of 
appropriately resourcing such work. Such resources include building in (and costing) sufficient time and expertise. They also include developing an adequate understanding amongst interdisciplinary research teams of the purpose of mixed methods research so that the implications of findings can be integrated as fully as possible. Such an understanding is only generated through deliberate strategies aimed at fostering team building, including developing trusting relationships. They also include developing the capacity on the part of qualitative researchers to understand the principles of RCT design and conduct - and the legislative frameworks within which such research operates.

Part of developing and nurturing such a research culture requires identifying and addressing the issues that still cause qualitative research to be marginalized within mainstream medical and health journals, and consequently continue to limit its contribution to research practice and evidence - most worrying given the need for mixed methods understanding and analysis in successful trial design and conduct. Only when there is a sufficient critical mass of crossdisciplinary understanding within the wider research community - including at the level of editorial boards and peer reviewers - can we genuinely re-conceptualize disciplinary boundaries and innovatively address the complexities of researching health. We suggest that such a re-conceptualization starts with a detailed and long-term strategic investment in research education at all levels across healthcare.

Authors' Note: EASE Back was reviewed and approved in the UK by the National Research Ethics Service Greater Manchester North Research Ethics Committee 12/NW/0227 and is registered with the current controlled trials database, ISRCTN, reference: ISRCTN49955124 
Acknowledgements: The EASE Back team includes Nadine Foster (Chief Investigator), Panos Barlas, Bernadette Bartlam, Annette Bishop, Melanie Holden, Khaled Ismail, Sue Jowett, Christine Kettle, Martyn Lewis, Alison Lloyd, Reuben Ogollah, Jackie Waterfield and Julie Young.

The team wish to thank the following staff of the Research Institute for Primary Care and Health Sciences: the administrative team (Michelle Robinson, Charlotte Purcell), IT support (Jo Smith, Wendy Clow), research management support (Helen Duffy, Ruth Beardmore, Liz Mason) and the physical therapy research facilitators (Tina Hadley-Barrows, Lucy Huckfield, Treena Larkin, Yvonne Rimmer, Julie Shufflebotham). The team also wish to thank the women who shared their experiences, the midwives and physical therapists who gave their time to advise and support the study, Hannah Cochrane for help in arranging the focus groups with physical therapists within the Stoke-On-Trent Partnership Trust Acupuncture Special Interest Group, and the NHS managers who allowed access to their staff teams.

Funding: The study was funded by the UK National Institute for Health Research (NIHR) under its Health Technology Assessment Programme (HTA) and also supported through an NIHR Research Professorship for NE Foster (NIHR-RP-011-015). The views expressed are those of the authors and not necessarily those of the NHS, the NIHR or the Department of Health.

Duel reporting: This paper focuses on exploring the role of qualitative research methods in clinical trials. In order to illustrate the arguments being made it has drawn on some of the research findings which have been outlined in the final report to the funding body (Foster et al. 2016).

Declaration of Conflicting Interests: The author(s) declared the following potential 
conflicts of interest with respect to the research, authorship, and/or publication of this article:

Dr Panos Barlas receives funding from training health professionals in acupuncture. 


\section{References}

Adler, N. E., \& Stewart, J. (2010). Using team science to address health disparities: MacArthur network as case example. Annals of the New York Academy of Sciences, 1186, 252-260. http://doi.org/10.1111/j.1749-6632.2009.05335.x

Arain, M., Campbell, M., Cooper, C., \& Lancaster, G. (2010). What is a pilot or feasibility study? A review of current practice and editorial policy. BMC Medical Research Methodology, 10(1), 67. Retrieved from http://www.biomedcentral.com/1471$2288 / 10 / 67$

Bartlam, B., Crome, P., Lally, F., Beswick, A.D., Cherubini, A., Clarfield, A.M., ... Zalewski, Z. (2012). The views of older people and carers on participation in clinical trials: the PREDICT Study. Journal of Clinical Investigation, vol. 2(3), 327-336.

Behrendt, C., Gölz, T., Roesler, C., Bertz, H., \& Wünsch, A. (2011). What do our patients understand about their trial participation? Assessing patients' understanding of their informed consent consultation about randomised clinical trials. Journal of Medical Ethics, 37(2), 74-80. Retrieved from http://search.ebscohost.com/login.aspx?direct=true\&AuthType=cookie,ip,url,uid,athens $\& \mathrm{db}=\mathrm{c} 8 \mathrm{~h} \& \mathrm{AN}=2011070952 \&$ site $=$ ehost-live

Bishop, A., Holden, M.A., Ogollah, R.O., \& Foster, N.E. 2015. Current management of pregnancy-related low back pain: a national cross-sectional survey of UK physiotherapists. Physiotherapy. pii: S0031-9406(15)03771-2. doi: 10.1016/j.physio.2015.02.003. [Epub ahead of print]

Bishop, A., Ogollah, R., Bartlam, B., Barlas, P., Ismail, K.M., Jowett, S., ... Foster, N.E. Under review. Evaluating Acupuncture and Standard care for pregnant womEn with BACK pain: The EASE Back pilot randomised controlled trial.

Bishop, F. L., Zaman, S., \& Lewith, G. T. (2011). Acupuncture for low back pain: a survey of clinical practice in the UK. Complementary Therapies in Medicine, 19(3), 144-8. http://doi.org/10.1016/j.ctim.2011.03.004

Bloom, B.S., Engelhart, M.D., Furst, E.J., Hill, W.H., \& Krathwohl, D. R. (1956). Taxonomy of educational objectives: The classification of educational goals. Handbook 1: Cognitive domain. New York: David McKay.

Bryman, A. (2007). Barriers to integrating quantitative and qualitative research. Journal of Mixed Methods Research, 1(1), 8-22. http://doi.org/10.1177/2345678906290531

Charmaz, K. (2006). Constructing grounded theory: A practical guide through qualitative analysis. London: Sage.

Cherubini, A., Oristrell, J., Pla, X, Ruggiero, C., Ferretti, R., Diestre, G., ... Mills, G.H. (2011). The persistent exclusion of older subjects from ongoing clinical trials on heart 
failure. Archives of Internal Medicine, 171(6), 550-556. Retrieved from http://archinte.jamanetwork.com/article.aspx?articleid=226912

Christ, T. W. (2007). A recursive approach to mixed methods research in a longitudinal Study of postsecondary education disability support services. Jounal of Mixed Methods Research, 1(3), 226-241.

Coakley, M., Fadiran, E. O., Parrish, L. J., Griffith, R. A., Weiss, E., \& Carter, C. (2012). Dialogues on diversifying clinical trials: successful strategies for engaging women and minorities in clinical trials. Journal Of Women's Health (2002), 21(7), 713-716. http://doi.org/10.1089/jwh.2012.3733

Curry, L. A., O’Cathain, A., Plano Clark, V. L., Aroni, R., Fetters, M., \& Berg, D. (2012). The role of group dynamics in mixed methods health sciences research teams. Journal of Mixed Methods Research, 6(1), 5-20. http://doi.org/10.1177/1558689811416941

Donovan, J., Mills, N., Smith, M., Brindle, L., Jacoby, A., Peters, T., Frankel, S., Neal, D., Hamdy, F. (2002). Quality improvement report: Improving design and conduct of randomised trials by embedding them in qualitative research: ProtecT (prostate testing for cancer and treatment) study. Commentary: presenting unbiased information to patients can be difficult. BMJ (Clinical Research Ed.), 325(7367), 766-770.

Ee, C. C., Manheimer, E., Pirotta, M. V, \& White, A. R. (2008). Acupuncture for pelvic and back pain in pregnancy: a systematic review. American Journal of Obstetrics and Gynecology, 198(3), 254-9. http://doi.org/10.1016/j.ajog.2007.11.008

Elden, H., Ostgaard, H.-C., Fagevik-Olsen, M., Ladfors, L., \& Hagberg, H. (2008). Treatments of pelvic girdle pain in pregnant women: adverse effects of standard treatment, acupuncture and stabilising exercises on the pregnancy, mother, delivery and the fetus/neonate. BMC Complementary and Alternative Medicine, 8(1), 34. http://doi.org/10.1186/1472-6882-8-34

European Union Research Advisory Board (2004). Interdisciplinarity in research, EURAB 04.009 - Final. On-line:

http://ec.europa.eu/research/eurab/pdf/eurab_04_009_interdisciplinarity_research_final. pdf

Foster, N.E., Bishop. A., Bartlam, B., Ogollah, R., Barlas, P., Holden, M.A., et al. (2016). Evaluating Acupuncture and Standard carE for pregnant women with Back pain (EASE Back): a feasibility study and pilot randomised trial. Health Technol Assess 20(33). doi: http://dx.doi.org/10.3310/hta20330

Freshwater, D. (2012). Managing movement, leading change. Journal of Mixed Methods Research, 6(1), 3-4. http://doi.org/10.1177/1558689812439873

Gagliardi, A. R., \& Dobrow, M. J. (2011). Paucity of qualitative research in general medical and health services and policy research journals: analysis of publication rates. BMC 
Health Services Research, 11, 268. http://doi.org/10.1186/1472-6963-11-268

Greenhalgh, T., Annandale, E., Ashcroft, R., Barlow, J., Black, N., Bleakley, A., ... Ziebland, S. (2016). An open letter to The BMJ editors on qualiative research. BMJ 352:i563. doi: http://dx.doi.org/10.1136/bmj.i563

Gray, B. (2008). Enhancing transdisciplinary research through collaborative leadership. American Journal of Preventive Medicine, 35(2), S124-32.

Hereu, P., Pérez, E., Fuentes, I., Vidal, X., Suñé, P., \& Arnau, J. M. (2010). Consent in clinical trials: What do patients know? Contemporary Clinical Trials, 31(5), 443-446. http://doi.org/10.1016/j.cct.2010.05.004

Howard, G., \& Howard, V. J. (2012). Observational epidemiology within randomized clinical trials: getting a lot for (almost) nothing. Progress in Cardiovascular Diseases, 54(4), 367-371. http://doi.org/10.1016/j.pcad.2011.08.003

Johnson, R. B., \& Onwuegbuzie, A. J. (2004). Mixed methods research : A research paradigm whose time has come. Educational Resrach.

Kessel, F., \& Rosenfield, P. L. (2008). Toward transdisciplinary research: historical and contemporary perspectives. American Journal of Preventive Medicine, 35(2), S225-34.

Kitzinger, J. (1994). The methodology of focus groups: the importance of interaction between research participants. The Sociology of Health and Illness: Critical Perspectives, 16(1), $103-121$.

Klassen, A. C., Creswell, J., Plano Clark, V. L., Smith, K. C., \& Meissner, H. I. (2012). Best practices in mixed methods for quality of life research. Quality of Life Research: An International Journal of Quality of Life Aspects of Treatment, Care \& Rehabilitation, 21(3), 377-380. http://doi.org/10.1007/s11136-012-0122-x

Leech, N.L., \& Onwuegbuzie, A. J. (2009). A typology of mixed methods research designs. Quality and Quantity, 43(2), 265-275.

Lewin, S., Glenton, C., \& Oxman, A. D. (2009). Use of qualitative methods alonside randomised controlled trials of complex healthcare interventisons: methodological study. British Medical Journal, 339(b3496). http://doi.org/10.1136/bmj.b3496

Mason, J. (2006). Mixing methods in a qualitatively driven way. Qualitative Research. http://doi.org/10.1177/1468794106058866

Mason, J., \& Dale, A. (2011). Creative tensions in social research: Questions of method. In A. Mason, J., Dale (Ed.), Understanding Social Research: Thinking Creatively About Method (pp. 1-26). London: Sage.

McBride, A. B. (2010). Toward a roadmap for interdisciplinary academic career success. Research \& Theory for Nursing Practice, 24(1), 74-86. http://doi.org/10.1891/15416577.24.1.74 
McDonald, A., Knight, R., Campbell, M., Entwistle, V., Grant, A., Cook, J., ... \& Snowdon, C. (2006). What influences recruitment to randomised controlled trials? A review of trials funded by two UK funding agencies. Trials, 7(1), 9.

Medical Research Council. (2008). Developing and evaluating complex interventions. Retrieved November 25, 2015, from https://www.mrc.ac.uk/documents/pdf/complexinterventions-guidance

Moher, D., Hopewell, S., Schulz, K. F., Montori, V., Gøtzsche, P. C., Devereaux, P. J., ... Altman, D. G. (2010). CONSORT 2010 Explanation and Elaboration: updated guidelines for reporting parallel group randomised trials. Journal of Clinical Epidemiology, 63(8), e1-e37. http://doi.org/10.1016/j.jclinepi.2010.03.004

Moore, G. F., Audrey, S., Barker, M., Bond, L., Bonell, C., Hardeman, W., .. Baird, J. (2015). Process evaluation of complex interventions: Medical Research Council guidance. Bmj. http://doi.org/10.1136/bmj.h1258

Morse, J. M., Barrett, M., Mayan, M., Olson, K., \& Spiers, J. (2008). Verification strategies for establishing reliability and validity in qualitative research. International Journal of Qualitative Methods, 1(2), 13-22.

National Institute for Clinical Excellence. (2009). Low back pain in adults: early management guidance and guidelines. Retrieved November 25, 2015, from https://www.nice.org.uk/guidance/cg88

O’Cathain, A., Murphy, E., \& Nicholl, J. (2008). Multidisciplinary, interdisciplinary, or dysfunctional? Team working in mixed-methods research. Qualitative Health Research, 18(11), 1574-1585. http://doi.org/10.1177/1049732308325535

O'Cathain, A., Murphy, E., \& Nicholl, J. (2010). Techniques designed to combine the results of qualitative and quantitative studies can provide researchers with more knowledge than separate analysis. British Medical Journal, 341. http://doi.org/http://dx.doi.org/10.1136/bmj.c4587

O’Cathain, A., Thomas, K. J., Drabble, S. J., Rudolph, A., \& Hewison, J. (2013). What can qualitative research do for randomised controlled trials? A systematic mapping review. BMJ Open, 3(6). http://doi.org/10.1136/bmjopen-2013-002889

O'Cathain, A., Hoddinott, P., Lewin, S., Thomas, K.J., Young, B., Adamson, J., ... Donovan, J. (2015). Maximising the impact of qualitative research in feasibility studies for randomised controlled trials: guidance for researchers. Pilot and Feasibility Studies, 1(32). http://doi.org/doi:10.1186/s40814-015-0026-y

Oakley, A. (2000). Experiments in knowing: gender and method in the social sciences. Cambridge, UK: Polity Press.

Olsson, C., Nilsson-Wikmar, L. (2004). Health-related quality of life and physical ability among pregnant women with and without back pain in late pregnancy. Acta Obstetricia 
et Gynecologica Scandinavica, 83(4), 351-357. http://doi.org/10.1111/j.00016349.2004.00384.x

Panel on New Directions in Social Demography, Social Epidemiology, and the Sociology of Aging. (2013). Committee on Population: Division on Behavioral and Social Sciences and Education, National Research Council: New directions in the sociology of aging, Waite, L.J. \& Plewes, T.J. editors. Washington: The National Academies Press. On-line: http://www.ncbi.nlm.nih.gov/books/NBK184362/

Pennick, V., \& Liddle, S. D. (2013). Interventions for preventing and treating pelvic and back pain in pregnancy. The Cochrane Database of Systematic Reviews, 8, CD001139. http://doi.org/10.1002/14651858.CD001139.pub3

Pierce, H., Homer, C. S. E., Dahlen, H. G., \& King, J. (2012). Pregnancy-related lumbopelvic pain: Listening to Australian women. Nursing Research and Practice, 2012, 1-10. http://doi.org/10.1155/2012/387428

Ross, S., Grant, A., Counsell, C., Gillespie, W., Russell, I., \& Prescott, R. (1999). Barriers to participation in randomised controlled trials: A Systematic Review. Journal of Clinical Epidemiology, 52(12), 1143-1156. http://doi.org/http://dx.doi.org/10.1016/S08954356(99)00141-9

Sim, J., \& Wright, C. (2000). Research in health care: Concepts, designs and methods. Cheltenham: Nelson Thornes.

Sully, B. G. O., Julious, S. A., \& Nicholl, J. (2013). A reinvestigation of recruitment to randomised, controlled, multicenter trials: a review of trials funded by two UK funding agencies. Trials, 14(1), 166. http://doi.org/10.1186/1745-6215-14-166

Tashakkori, A., \& Creswell, J. W. (2007). Editorial: Exploring the nature of research questions in mixed methods research. Journal of Mixed Methods Research, 1(3), 207211. http://doi.org/10.1177/1558689807302814

Thomas, K.J., MacPherson, H., Ratcliffe, J., Thorpe, L., Brazier, J., Campbell, M., ... Nicholl, J. P. (2005). Longer term clinical and economic benefits of offering acupuncture care to patients with chronic low back pain. Health Technology Assessment (Winchester, England), 9(32), iii-iv, ix-x, 1-109.

Tickle-Degnen, L. (2013). Nuts and bolts of conducting feasibility studies. The American Journal of Occupational Therapy, 67(2), 6. http://doi.org/http://doi.org/10.5014/ajot.2013.006270

Vermani, E., Mittal, R., \& Weeks, A. (2010). Pelvic girdle pain and low back pain in pregnancy: a review. Pain Practice: The Official Journal of World Institute of Pain, 10(1), 60-71. http://doi.org/10.1111/j.1533-2500.2009.00327.x

Waterfield, J., Bartlam, B., Holden, M.A., Bishop, A., Barlas, P., Foster, N.E. 2015. Physical therapists' views and experiences of pregnancy-related low back pain and the role of 
acupuncture: a qualitative exploration. Physical Therapy 95(9): 1234-1243; doi:

10.2522/ptj.20140298

Wilkinson, J., Powell, A., \& Davies, H. (2011). Healthcare professionals' views on clinician engagement in quality improvement. London. Retrieved from http://www.health.org.uk/publication/healthcare-professionals\%E2\%80\%99-viewsclinician-engagement-quality-improvement

Wisdom, J. P., Cavaleri, M. A., Onwuegbuzie, A. J., \& Green, C. A. (2012). Methodological reporting in qualitative, quantitative, and mixed methods health services research articles. Health Services Research, 47(2), 721-745. http://doi.org/10.1111/j.14756773.2011.01344.x

Wu, W. H., Meijer, O. G., Uegaki, K., Mens, J. M. A., van Dieën, J. H., Wuisman, P. I. J. M., \& Östgaard, H. C. (2004). Pregnancy-related pelvic girdle pain (PPP), I: Terminology, clinical presentation, and prevalence. European Spine Journal, 13(7), 575-589. http://doi.org/10.1007/s00586-003-0615-y 
Table 1: Summary of participants in qualitative research $n=53$

Table 1: Summary of Interview and Focus Group participants $n=53$

\begin{tabular}{|c|c|c|c|}
\hline Participants & Individual interviews & Focus groups & $n$ \\
\hline $\begin{array}{l}\text { Pregnant } \\
\text { women }\end{array}$ & 17 & 0 & 17 \\
\hline Midwives & 0 & $\begin{array}{l}3: \\
2 \times \text { community midwives } \\
1 \times \text { research midwives }\end{array}$ & 15 \\
\hline $\begin{array}{l}\text { Physical } \\
\text { therapists }\end{array}$ & 3 & $\begin{array}{l}3: \\
2 \times \text { community physical therapists } \\
1 \times \text { women's health physical therapists }\end{array}$ & 18 \\
\hline Total & & & 53 \\
\hline
\end{tabular}

Table 2: Summary of referral sources for of women with pregnancy-related LBP

\begin{tabular}{|l|l|}
\hline Referral Source & Numbers Referred \\
\hline Self-referred & 2 \\
\hline Physical Therapists & 2 \\
\hline Obstetricians & 3 \\
\hline Midwives & 20 \\
\hline Back class & 16 \\
\hline Internet & 0 \\
\hline Total & $\mathbf{4 3}$ \\
\hline
\end{tabular}


Figure 1: Question-driven mixed methods process model in EASE Back

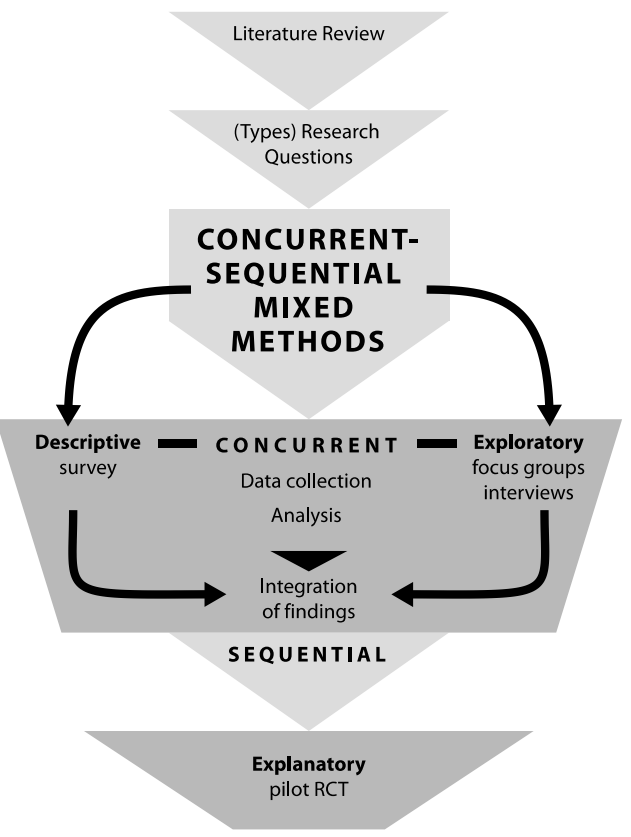

